\title{
Article \\ Systems of Simultaneous Differential Inclusions Implying Function Containment
}

\author{
José A. Antonino ${ }^{1}$ and Sanford S. Miller ${ }^{2, *}$ \\ 1 Departamento de Matemática Aplicada, ETSICCP, Universidad Politécnica de Valencia, 46071 Valencia, Spain; \\ jantonin@mat.upv.es \\ 2 Department of Mathematics, SUNY Brockport, Brockport, NY 14420, USA \\ * Correspondence: smiller@brockport.edu
}

Citation: Antonino, J.A.; Miller, S.S. Systems of Simultaneous Differential Inclusions Implying Function Containment. Mathematics 2021, 9 , 1252. https://doi.org/10.3390/ math9111252

Academic Editor: Georgia Irina Oros

Received: 18 January 2021

Accepted: 24 May 2021

Published: 30 May 2021

Publisher's Note: MDPI stays neutral with regard to jurisdictional claims in published maps and institutional affiliations.

Copyright: (c) 2021 by the authors. Licensee MDPI, Basel, Switzerland. This article is an open access article distributed under the terms and conditions of the Creative Commons Attribution (CC BY) license (https:// creativecommons.org/licenses/by/ $4.0 /)$.

\begin{abstract}
An important problem in complex analysis is to determine properties of the image of an analytic function $p$ defined on the unit disc $\mathbf{U}$ from an inclusion or containment relation involving several of the derivatives of $p$. Results dealing with differential inclusions have led to the development of the field of Differential Subordinations, while results dealing with differential containments have led to the development of the field of Differential Superordinations. In this article, the authors consider a mixed problem consisting of special differential inclusions implying a corresponding containment of the form $D[p](\mathbf{U}) \subset \Omega \Rightarrow \Delta \subset p(\mathbf{U})$, where $\Omega$ and $\Delta$ are sets in $\mathbb{C}$, and $D$ is a differential operator such that $D[p]$ is an analytic function defined on $\mathbf{U}$. We carry out this research by considering the more general case involving a system of two simultaneous differential operators in two unknown functions.
\end{abstract}

Keywords: differential inclusions; differential containments; differential inequalities; differential subordinations; univalent functions

MSC: primary 34A40; 34A60; secondary 30C 80

\section{Introduction}

We begin by introducing the important classes of functions considered in this article. Let $\mathcal{H}=\mathcal{H}[\mathbf{U}]$ denote the class of functions analytic in the unit disk $\mathbf{U}$, and let

$$
\mathcal{H}[a, n]=\left\{f \in \mathcal{H}: f(z)=a+a_{n} z^{n}+\cdots\right\} .
$$

A common problem in complex analysis is to determine the range of a function $p \in \mathcal{H}[a, n]$ from a differential inclusion or containment relation involving several of the derivatives of $p$. Let $\Omega$ and $\Delta$ be sets in $\mathbb{C}$, and $D$ be a differential operator such that $D[p]$ is an analytic function defined on $\mathbf{U}$. A natural question is to ask what conditions on $D$, $\Omega$ and $\Delta$ are needed so that

$$
D[p](\mathbf{U}) \subset \Omega \Rightarrow p(\mathbf{U}) \subset \Delta .
$$

In this case, we have a differential inclusion $\Rightarrow$ function inclusion. There are many papers of this type that deal with special differential inclusions implying an inclusion for the image of the function $p$. Similarly, there are many papers that deal with special differential containments and corresponding containments for the image of the function $p$ of the form

$$
\Omega \subset D[p](\mathbf{U}) \Rightarrow \Delta \subset p(\mathbf{U}) .
$$

In this case, we have a differential containment $\Rightarrow$ function containment. Both sets of papers have resulted in many applications in complex analysis. See the monographs [1,2] for many results, applications and extensive bibliographies of results such as (1) and (2). 
An open question to consider is to combine the two concepts in (1) and (2) and determine conditions on $D, \Omega$ and $\Delta$ so that the mixed problem of differential inclusions implies a function containment of the form

$$
D[p](\mathbf{U}) \subset \Omega \Rightarrow \Delta \subset p(\mathbf{U}) .
$$

In this case, we have a differential inclusion $\Rightarrow$ function containment.

In a recent article [3] the authors have extended results described in (1) to systems of two simultaneous second-order differential operators in two complex-valued functions. It is our intention to do the same with (3).

\section{Definitions}

We first indicate the forms of the two simultaneous second-order analytic differential operators that we will consider in this article.

Definition 1. Let $D_{i}: \mathbb{C}^{7} \rightarrow \mathbb{C}$ and let $\lambda_{i}(z)$ be analytic in $\boldsymbol{U}$ for $i=1,2$. For $p \in \mathcal{H}[a, n]$ and $q \in \mathcal{H}[b, n]$ we define the second-order differential operators $D_{i}\left[p, q, \lambda_{i}\right]$, for $i=1,2, b y$

$$
D_{i}\left[p, q, \lambda_{i}\right](z) \equiv D_{i}\left[p(z), z p^{\prime}(z), z^{2} p^{\prime \prime}(z), q(z), z q^{\prime}(z), z^{2} q^{\prime \prime}(z), \lambda_{i}(z)\right]
$$

Throughout this article we will assume that $D_{i}\left[p, q, \lambda_{i}\right]$ is analytic in $\boldsymbol{U}$.

Let $\Omega_{i}$ and $\Delta_{i}$ be sets in $\mathbb{C}$ and $D_{i}\left[p, q, \lambda_{i}\right]$ be the second-order differential operators defined in (4), for $i=1,2$. The analogue of (3) that we will consider in this article deals with two simultaneous differential inclusions implying function containments of the following form

$$
\left\{\begin{array} { l } 
{ D _ { 1 } [ p , q , \lambda _ { 1 } ] ( \mathbf { U } ) \subset \Omega _ { 1 } } \\
{ D _ { 2 } [ p , q , \lambda _ { 2 } ] ( \mathbf { U } ) \subset \Omega _ { 2 } }
\end{array} \quad \Longrightarrow \quad \left\{\begin{array}{l}
\Delta_{1} \subset p(\mathbf{U}) \\
\Delta_{2} \subset q(\mathbf{U})
\end{array} .\right.\right.
$$

In many cases, the containments on the right-sides of (5) can be written in terms of superordinations. We recall those definitions. Let $f$ and $F$ be members of $\mathcal{H}$. The function $f$ is said to be subordinate to $F$ (or $F$ is superordinate to $f$ ), written $f \prec F$, if there exists a function $w$ analytic in $\mathbf{U}$ with $w(0)=0$ and $|w(z)|<1$, such that $f(z)=F(w(z))$. If, in addition, $F$ is univalent, then $f \prec F$ if and only if $f(0)=F(0)$ and $f(\mathbf{U}) \subset F(\mathbf{U})$.

If $p$ and $q$ in (5) are univalent, and $\Delta_{1}$ and $\Delta_{2}$ are simply connected domains, then it is possible to rephrase the right-side of (5) in terms of superordination. If $\Delta_{1}$ is a simply connected domain containing the point $p(0)=a$ and $\Delta_{1} \neq \mathbb{C}$, then there is a conformal mapping $g_{1}$ of $\mathbf{U}$ onto $\Delta_{1}$ such that $g_{1}(0)=a$, and if $\Delta_{2}$ is a simply connected domain containing the point $q(0)=b$ and $\Delta_{2} \neq \mathbb{C}$, then there is a conformal mapping $g_{2}$ of $\mathbf{U}$ onto $\Delta_{2}$ such that $g_{2}(0)=b$. In this case, (5) can be rewritten as

$$
\left\{\begin{array} { l } 
{ D _ { 1 } [ p , q , \lambda _ { 1 } ] ( \mathbf { U } ) \subset \Omega _ { 1 } } \\
{ D _ { 2 } [ p , q , \lambda _ { 2 } ] ( \mathbf { U } ) \subset \Omega _ { 2 } }
\end{array} \quad \Longrightarrow \quad \left\{\begin{array}{l}
g_{1}(z) \prec p(z) \\
g_{2}(z) \prec q(z)
\end{array} .\right.\right.
$$

We shall refer to the left sides of (5) and (6) as a System of Simultaneous Differential Inclusions (SSDI).

There are three basic pairs of elements in (5) and (6): the differential operators $D_{i}$, the sets $\Omega_{i}$, and the sets $\Delta_{i}$ (or functions $g_{i}$ ). If two of these elements are given, one would hope to find conditions on the third.

Our aim in this article is to solve a system of such simultaneous differential inclusionsanalogous to solving a system of simultaneous differential equations in the real-plane. We restrict our development to systems consisting of two second-order differential inclusions in two unknown functions. The results presented here can be extended in a natural way to their corresponding third-order cases. We begin by introducing some important definitions. 
Definition 2. Let $\Omega_{i}$ be sets in $\mathbb{C}$ and let $D_{i}\left[p, q, \lambda_{i}\right]$ be the analytic differential operators defined in (4) for $i=1$, 2. If $p \in \mathcal{H}[a, n]$ and $q \in \mathcal{H}[b, n]$ satisfy the SSDI

$$
\left\{\begin{array}{l}
D_{1}\left[p, q, \lambda_{1}\right](\boldsymbol{U}) \subset \Omega_{1} \\
D_{2}\left[p, q, \lambda_{2}\right](\boldsymbol{U}) \subset \Omega_{2}
\end{array},\right.
$$

then $p$ and $q$ are called Solutions of the SSDI.

We will show that certain SSDI's have solutions, and that these solutions have particular properties such as those given on the right-sides of (5) and (6).

Example 1. Let $p \in \mathcal{H}[0,1]$ and $q \in \mathcal{H}[0,1]$ and consider the SSDI given by

$$
\left\{\begin{array}{l}
\left\{\begin{array}{r}
\left.-z p^{\prime}(z)+2 q(z): z \in \boldsymbol{U}\right\} \subset \boldsymbol{U} \\
\left.2 p(z)-z q^{\prime}(z): z \in \boldsymbol{U}\right\} \subset \boldsymbol{U}
\end{array} .\right.
\end{array}\right.
$$

It is easy to check that the univalent functions $p(z)=q(z)=z+z^{2} / 2$ are Solutions of the SSDI given in (8).

Example 2. Let $\Omega_{i}=\{z: \operatorname{Re} z>0\}$, the right half plane for $i=1$,2. Let $p \in \mathcal{H}[0,1]$ and $q \in \mathcal{H}[0,1]$ and consider the SSDI given by

$$
\left\{\begin{array}{l}
\left\{-z p^{\prime}(z)+2 z q^{\prime}(z): z \in \boldsymbol{U}\right\} \subset \Omega_{1} \\
\left\{z^{2} p^{\prime \prime}(z)-5 z q^{\prime}(z): z \in \boldsymbol{U}\right\} \subset \Omega_{2}
\end{array} .\right.
$$

It is clear that this SSDI has no solutions since there are no analytic functions $p$ and $q$ that can satisfy this system at $z=0$.

Definition 3. The set of analytic functions $\left\{g_{1}, g_{2}\right\}$ as given in (6) is called a set of subordinants of the Solutions of the SSDI (6) or more simply a set of subordinants if $g_{1} \prec p$ and $g_{2} \prec q$ for all $p$ and $q$ satisfying the left-side of (6). A set of subordinants $\left\{\tilde{g}_{1}, \tilde{g}_{2}\right\}$ that satisfies $g_{1} \prec \tilde{g}_{1}$ and $g_{2} \prec \tilde{g}_{2}$ for all subordinants $\left\{g_{1}, g_{2}\right\}$ of (6) is called a set of best subordinants of (6). Please note that the set of best subordinants is unique up to a rotation of $\boldsymbol{U}$.

It is our intent to show that for certain types of SSDI we can obtain corresponding sets of subordinants and best subordinants $\left\{\tilde{g}_{1}, \tilde{g}_{2}\right\}$ of the system.

The analogue of the best subordinants in Definition 3 for the SSDI (5) would be finding the largest inclusion sets $\tilde{\Delta}_{1}$ and $\tilde{\Delta}_{2}$ such that

$$
\left\{\begin{array}{lll}
D_{1}\left[p, q, \lambda_{1}\right](\mathbf{U}) \subset \Omega_{1} & \Rightarrow & \tilde{\Delta}_{1} \subset p(\mathbf{U}) \\
D_{2}\left[p, q, \lambda_{2}\right](\mathbf{U}) \subset \Omega_{2} & \Rightarrow & \tilde{\Delta}_{2} \subset q(\mathbf{U})
\end{array} .\right.
$$

\section{Admissibility and a Fundamental Theorem}

For the development of the theory we need to the consider the following class of univalent functions defined on the closed unit disc.

Definition 4. Let $Q$ denote the set of functions $g$ that are analytic and univalent on the set $\overline{\boldsymbol{u}} \backslash \boldsymbol{E}(\mathrm{g})$, where

$$
E(g)=\left\{\zeta \in \partial U: \lim _{z \rightarrow \zeta} g(z)=\infty\right\}
$$

and are such that $\operatorname{Min}\left|g^{\prime}(\zeta)\right|=\rho>0$ for $\zeta \in \partial \boldsymbol{U} \backslash \boldsymbol{E}(\mathrm{g})$. The subclass of $\boldsymbol{Q}$ for which $g(0)=a$ is denoted by $Q(a)$. 
As a simple example of a member of the class $Q(1)$, consider the function $g(z)=$ $(1+z) /(1-z)$. For this function we have $\boldsymbol{E}(g)=\{1\}$, and $\operatorname{Min}\left|g^{\prime}(\zeta)\right|=1 / 2>0$ for $\zeta \in \partial \mathbf{U} \backslash\{1\}$ and hence $g \in Q(1)$.

The following lemma [1] (p. 22) and [4] has played a key role in many results involving the theory of differential subordinations and will also play a key role in this article.

Lemma 1 (Miller/Mocanu Lemma.). Let $q \in \mathcal{H}[a, n]$ with $q(z) \not \equiv a$ and $n \geq 1$, and let $p \in \boldsymbol{Q}(a)$. If there exist points $z_{0}=r_{0} e^{i \theta_{0}} \in \boldsymbol{U}$ and $\zeta_{0} \in \partial \boldsymbol{U} \backslash \boldsymbol{E}(p)$ such that $q\left(z_{0}\right)=p\left(\zeta_{0}\right)$, and $q\left(\boldsymbol{U}_{r_{0}}\right) \subset p(\boldsymbol{U})$, then there exists an $m$, where $m \geq n \geq 1$ such that

$$
\zeta_{0} p^{\prime}\left(\zeta_{0}\right)=z_{0} q^{\prime}\left(z_{0}\right) / m \text { and } \operatorname{Re} \frac{\zeta_{0} p^{\prime \prime}\left(\zeta_{0}\right)}{p^{\prime}\left(\zeta_{0}\right)}+1 \leq \frac{1}{m}\left[\operatorname{Re} \frac{z_{0} q^{\prime \prime}\left(z_{0}\right)}{q^{\prime}\left(z_{0}\right)}+1\right] .
$$

We first define a special class of differential operators needed to solve a SSDI.

Definition 5. Let $\lambda_{i}$ be analytic in $\overline{\boldsymbol{U}}$, and $g_{i} \in Q$ with corresponding sets $\boldsymbol{E}\left(g_{i}\right)$ as given in Definition 4 for $i=1$, 2 . Let $\left(\Omega_{1}, \Omega_{2}\right)$ be a subset of $\mathbb{C} \times \mathbb{C}$ and let $n_{1}$ and $n_{2}$ be positive integers. The Set of Admissible Differential Operators $\boldsymbol{\Psi}_{\left(n_{1}, n_{2}\right)}\left[\left(\lambda_{1}, \lambda_{2}\right),\left(\Omega_{1}, \Omega_{2}\right),\left(g_{1}, g_{2}\right)\right]$ consists of those pairs of differential operators $\left(D_{1}, D_{2}\right)$, with $D_{i}: \mathbb{C}^{7} \rightarrow \mathbb{C}$ as given in Definition 1 , for $i=1,2$, which satisfy the two admissibility conditions

$$
D_{1}\left[r, s, t, g_{2}(\zeta), \zeta g_{2}^{\prime}(\zeta), \zeta^{2} g_{2}^{\prime \prime}(\zeta), \lambda_{1}(\zeta)\right] \notin \bar{\Omega}_{1}
$$

when $r=g_{1}(z), s=z g_{1}^{\prime}(z) / m_{1}, \operatorname{Re} \frac{t}{s}+1 \leq \frac{1}{m_{1}} \operatorname{Re}\left[\frac{z g_{1}^{\prime \prime}(z)}{g_{1}^{\prime}(z)}+1\right]$,

$z \in \mathbf{U}, \zeta \in \partial \mathbf{U} \backslash \boldsymbol{E}\left(g_{2}\right)$ and $m_{1} \geq n_{1} \geq 1$.

$$
D_{2}\left[g_{1}(\eta), \eta g_{1}^{\prime}(\eta), \eta^{2} g_{1}^{\prime \prime}(\eta), \rho, \sigma, \tau, \lambda_{2}(\eta)\right] \notin \bar{\Omega}_{2}
$$

when $\rho=g_{2}(z), \sigma=z g_{2}^{\prime}(z) / m_{2}, \operatorname{Re} \frac{\tau}{\sigma}+1 \leq \frac{1}{m_{2}} \operatorname{Re}\left[\frac{z g_{2}^{\prime \prime}(z)}{g_{2}^{\prime}(z)}+1\right]$,

$z \in \mathbf{U}, \eta \in \partial \mathbf{U} \backslash \boldsymbol{E}\left(g_{1}\right)$ and $m_{2} \geq n_{2} \geq 1$.

In the special case when $n_{1}=n_{2}=1$, we denote the set of operators $\Psi_{(1,1)}\left[\left(\lambda_{1}, \lambda_{2}\right)\right.$, $\left.\left(\Omega_{1}, \Omega_{2}\right),\left(g_{1}, g_{2}\right)\right]$ by $\boldsymbol{\Psi}\left[\left(\lambda_{1}, \lambda_{2}\right),\left(\Omega_{1}, \Omega_{2}\right),\left(g_{1}, g_{2}\right)\right]$. In the special case when $\Omega_{1} \neq \mathbb{C}$ and $\Omega_{2} \neq \mathbb{C}$ are simply connected domains and $h_{1}$ and $h_{2}$ are conformal maps of $\mathbf{U}$ onto $\Omega_{1}$ and $\Omega_{2}$ respectively, we denote the set $\boldsymbol{\Psi}_{\left(n_{1}, n_{2}\right)}\left[\left(\lambda_{1}, \lambda_{2}\right),\left(h_{1}(U), h_{2}(U)\right),\left(g_{1}, g_{2}\right)\right]$ by $\boldsymbol{\Psi}_{\left(n_{1}, n_{1}\right)}\left[\left(\lambda_{1}, \lambda_{2}\right),\left(h_{1}, h_{2}\right),\left(g_{1}, g_{2}\right)\right]$.

In the case of first-order differential operators the admissibility conditions (9) and (10), with $D_{i}: \mathbb{C}^{5} \rightarrow \mathbb{C}$ for $i=1,2$ simplify to

$$
D_{1}\left[g_{1}(z), z g_{1}^{\prime}(z) / m_{1}, g_{2}(\zeta), \zeta g_{2}^{\prime}(\zeta), \lambda_{1}(\zeta)\right] \notin \bar{\Omega}_{1}
$$

when $z \in \mathbf{U}, \zeta \in \partial \mathbf{U} \backslash \boldsymbol{E}\left(g_{2}\right)$ and $m_{1} \geq n_{1} \geq 1$.

$$
D_{2}\left[g_{1}(\eta), \eta g_{1}^{\prime}(\eta), g_{2}(z), z g_{2}^{\prime}(z) / m_{2}, \lambda_{2}(\eta)\right] \notin \bar{\Omega}_{2}
$$

when $z \in \mathbf{U}, \eta \in \partial \mathbf{U} \backslash \boldsymbol{E}\left(g_{1}\right)$ and $m_{2} \geq n_{2} \geq 1$.

A closer look at conditions (9) and (10) [or (11) and (12)] indicate that there are different conditions on each of the operators $D_{1}$ and $D_{2}$ in the pair $\left(D_{1}, D_{2}\right)$. An operator pair $\left(D_{\alpha}, D_{\beta}\right)$ may not be in the Set of Admissible Operators as given by Definition 5 , but the pair $\left(D_{\beta}, D_{\alpha}\right)$ may be in the Set of Admissible Operators. We will see a case of this in Examples 3 and 4 . In Example 3 we show that the pair $\left(D_{\alpha}, D_{\beta}\right)$ is not in the Set of Admissible Operators, while in Example 4 we show that the pair $\left(D_{\beta}, D_{\alpha}\right)$ is in the Set of Admissible Operators. 
Example 3. Let $\Omega=\Omega_{1}=\Omega_{2}=\{\operatorname{Re} z>0: z \in \boldsymbol{U}\}$, the right-half complex plane, and let $p \in \mathcal{H}\left[a, n_{1}\right] \cap \boldsymbol{Q}(a)$ and $q \in \mathcal{H}\left[b, n_{2}\right] \cap \boldsymbol{Q}(b)$ satisfy the SSDI

$$
\left\{\begin{array}{l}
D_{1}\left[p, q, \lambda_{1}\right](\boldsymbol{U}) \equiv\left\{2 p(z)+z p^{\prime}(z)-q(z): z \in \boldsymbol{U}\right\} \subset \Omega \\
D_{2}\left[p, q, \lambda_{2}\right](\boldsymbol{U}) \equiv\left\{q(z)+z q^{\prime}(z): z \in \boldsymbol{U}\right\} \subset \Omega
\end{array} .\right.
$$

We will show that this pair $\left(D_{1}, D_{2}\right)$ with the functions $g_{1}(z)=g_{2}(z)=(1+z) /(1-z)$ is not in the Set of Admissible Operators. Writing (13) in standard form we see that the functions $D_{1}$ and $D_{2}$ are of the form

$$
\left\{\begin{array}{l}
D_{1}\left[r, s, t, \rho, \sigma, \tau, \lambda_{1}(z)\right]=2 r+s-\rho \\
D_{2}\left[r, s, t, \rho, \sigma, \tau, \lambda_{2}(z)\right]=\rho+\sigma
\end{array} .\right.
$$

We need to show that this pair of operators does not satisfy

$$
\left(D_{1}, D_{2}\right) \in \boldsymbol{\Psi}_{\left(n_{1}, n_{1}\right)}\left[(0,0),\left(\Omega_{1}, \Omega_{2}\right),\left(g_{1}, g_{2}\right)\right] .
$$

In order for this last statement to be true, according to condition (9) of the first part of Definition 5, requires showing that

$$
2 g_{1}(z)+z g_{1}^{\prime}(z) / m_{1}-\frac{1+\zeta}{1-\zeta} \notin \bar{\Omega},
$$

when $z \in \mathbf{U}, \zeta \in \partial \mathbf{U} \backslash \boldsymbol{E}\left(g_{2}\right)$ and $m_{1} \geq n_{1} \geq 1$. This condition is equivalent to requiring that

$$
\operatorname{Re}\left[2 \frac{1+z}{1-z}+\frac{2 z}{(1-z)^{2} m_{1}}-\frac{1+\zeta}{1-\zeta}\right]<0
$$

Since this is not satisfied when $z=0$, condition (14) cannot be satisfied and the pair of differential operators given in (13) is not in the Set of Admissible Operators.

We next interchange the differential operators in Example 3 to obtain an appropriate pair of operators.

Example 4. Let $\Omega=\Omega_{1}=\Omega_{2}=\{\operatorname{Re} z>0: z \in U\}$, the right-half complex plane, and let $p \in \mathcal{H}\left[a, n_{1}\right] \cap \boldsymbol{Q}(a)$ and $q \in \mathcal{H}\left[b, n_{2}\right] \cap \boldsymbol{Q}(b)$ satisfy the SSDI

$$
\left\{\begin{array}{l}
D_{1}\left[p, q, \lambda_{1}\right](\boldsymbol{U}) \equiv\left\{q(z)+z q^{\prime}(z): z \in \boldsymbol{U}\right\} \subset \Omega \\
D_{2}\left[p, q, \lambda_{2}\right](\boldsymbol{U}) \equiv\left\{2 p(z)+z p^{\prime}(z)-q(z): z \in \boldsymbol{U}\right\} \subset \Omega
\end{array} .\right.
$$

We will show that this pair $\left(D_{1}, D_{2}\right)$, with the functions $g_{1}(z)=g_{2}(z)=(1+z) /(1-z)$, is in the Set of Admissible Operators. Writing (15) in standard form we see that the functions $D_{1}$ and $D_{2}$ are of the form

$$
\left\{\begin{array}{l}
D_{1}\left[r, s, t, \rho, \sigma, \tau, \lambda_{1}(z)\right]=\rho+\sigma \\
D_{2}\left[r, s, t, \rho, \sigma, \tau, \lambda_{2}(z)\right]=2 r+s-\rho
\end{array} .\right.
$$

We need to show that $\left(D_{1}, D_{2}\right) \in \boldsymbol{\Psi}_{\left(n_{1}, n_{1}\right)}\left[(0,0),\left(\Omega_{1}, \Omega_{2}\right),\left(g_{1}, g_{2}\right)\right]$. According to Definition 5 , we need to show that

$$
\begin{aligned}
& g_{2}(\zeta)+\zeta g_{2}^{\prime}(\zeta) \notin \bar{\Omega} \\
& 2 g_{1}(\eta)+\eta g_{1}^{\prime}(\eta)-g_{2}(z) \notin \bar{\Omega}
\end{aligned}
$$

when $z \in \mathbf{U}, \zeta \in \partial \mathbf{U} \backslash \boldsymbol{E}\left(g_{2}\right)$ and $\eta \in \partial \mathbf{U} \backslash \boldsymbol{E}\left(g_{1}\right)$. This follows since 


$$
\begin{aligned}
& \operatorname{Re}\left[g_{2}(\zeta)+\zeta g_{2}^{\prime}(\zeta)\right]=\operatorname{Re}\left[\frac{1+\zeta}{1-\zeta}+\frac{2 \zeta}{(1-\zeta)^{2}}\right]<0 \text { and } \\
& \operatorname{Re}\left[2 g_{1}(\eta)+\eta g_{1}^{\prime}(\eta)-g_{2}(z)\right]=\operatorname{Re}\left[2 \frac{1+\eta}{1-\eta}+\frac{2 \eta}{(1-\eta)^{2}}-\frac{1+z}{1-z}\right]<0 .
\end{aligned}
$$

Hence $\left(D_{1}, D_{2}\right)$ is in the Set of Admissible Operators.

The following theorem is a foundation result for the theory of Second-Order SSDI.

Theorem 1. Let $\Omega_{1}$ and $\Omega_{2}$ be sets in $\mathbb{C}$, let $\lambda_{1}$ and $\lambda_{2}$ be analytic in $\overline{\boldsymbol{U}}$, let $g_{1} \in Q(a), g_{2} \in Q(b)$, and let $\left(D_{1}, D_{2}\right) \in \Psi_{\left(n_{1}, n_{2}\right)}\left[\left(\lambda_{1}, \lambda_{2}\right),\left(\Omega_{1}, \Omega_{2}\right),\left(g_{1}, g_{2}\right)\right]$. If $p \in \mathcal{H}\left[a, n_{1}\right] \cap Q(a)$ and $q \in$ $\mathcal{H}\left[b, n_{2}\right] \cap \boldsymbol{Q}(b)$ satisfy the SSDI

$$
\left\{\begin{array}{l}
D_{1}\left[p, g_{2}, \lambda_{1}\right](\boldsymbol{U}) \subset \Omega_{1} \\
D_{2}\left[g_{1}, q, \lambda_{2}\right](\boldsymbol{U}) \subset \Omega_{2}
\end{array}\right.
$$

then $g_{1} \prec p$ and $g_{2} \prec q$, and $\left\{g_{1}, g_{2}\right\}$ are a set of subordinants of (16).

Proof. (a) For the first implication, if we assume $g_{1} \nprec p$, then by Lemma 1 there exist points $z_{0}=r_{0} e^{i \theta_{0}} \in \mathbf{U}, \zeta_{0} \in \partial \mathbf{U} \backslash E\left(g_{1}\right)$ and $m_{1} \geq n_{1} \geq 1$ such that $g_{1}\left(z_{0}\right)=p\left(\zeta_{0}\right)$, $g_{1}\left(U_{r_{0}}\right) \subset p(U)$,

$$
\zeta_{0} p^{\prime}\left(\zeta_{0}\right)=z_{0} g_{1}^{\prime}\left(z_{0}\right) / m_{1} \text { and } \operatorname{Re} \frac{\zeta_{0} p^{\prime \prime}\left(\zeta_{0}\right)}{p^{\prime}\left(\zeta_{0}\right)}+1 \leq \frac{1}{m_{1}}\left[\operatorname{Re} \frac{z_{0} g_{1}^{\prime \prime}\left(z_{0}\right)}{g_{1}^{\prime}\left(z_{0}\right)}+1\right] .
$$

Using these results in (9) of Definition 5 we conclude

$$
D_{1}\left[p\left(\zeta_{0}\right), \zeta_{0} p^{\prime}\left(\zeta_{0}\right), \zeta_{0}^{2} p^{\prime \prime}\left(\zeta_{0}\right), g_{2}\left(\zeta_{0}\right), \zeta_{0} g_{2}^{\prime}\left(\zeta_{0}\right), \zeta_{0}^{2} g_{2}^{\prime \prime}\left(\zeta_{0}\right), \lambda_{1}\left(\zeta_{0}\right)\right] \notin \bar{\Omega}_{1} .
$$

Since this contradicts the first part of (16) we must have $g_{1} \prec p$.

(b) For the second implication, if we assume $g_{2} \nprec q$, then by Lemma 1 there exist points $z_{0}=r_{0} e^{i \theta_{0}} \in \mathbf{U}, \eta_{0} \in \partial \mathbf{U} \backslash E\left(g_{2}\right)$ and $m_{2} \geq n_{2} \geq 1$ such that $g_{2}\left(z_{0}\right)=q\left(\eta_{0}\right)$, $g_{2}\left(U_{r_{0}}\right) \subset q(U)$,

$$
\eta_{0} q^{\prime}\left(\eta_{0}\right)=z_{0} g_{2}^{\prime}\left(z_{0}\right) / m_{2} \text { and } \operatorname{Re} \frac{\eta_{0} q^{\prime \prime}\left(\eta_{0}\right)}{q^{\prime}\left(\eta_{0}\right)}+1 \leq \frac{1}{m_{2}}\left[\operatorname{Re} \frac{z_{0} g_{2}^{\prime \prime}\left(z_{0}\right)}{g_{2}^{\prime}\left(z_{0}\right)}+1\right] .
$$

Using these results in (10) of Definition 5 we obtain

$$
D_{2}\left[g_{1}\left(\eta_{0}\right), \eta_{0} g_{1}^{\prime}\left(\eta_{0}\right), \eta_{0}^{2} g_{1}^{\prime \prime}\left(\eta_{0}\right), q\left(\eta_{0}\right), \eta_{0} q^{\prime}\left(\eta_{0}\right), \eta_{0}{ }^{2} q^{\prime \prime}\left(\eta_{0}\right), \lambda_{2}\left(\eta_{0}\right)\right] \notin \bar{\Omega}_{2} .
$$

Since this contradicts the second part of (16) we must have $g_{2} \prec q$.

As a result of the above theorem we can obtain subordinants of a SSDI of the form (16) by merely checking that the operators $D_{1}$ and $D_{2}$ satisfy the admissibility conditions (9) and (10) [or (11) and (12)] of Definition 5. This simple algebraic check yields subordinants of various SSDI that would be very difficult to obtain directly.

In the following two examples we use Theorem 1 to find subordinants of a SSDI.

Example 5. Let $\boldsymbol{U}_{r}=\{z:|z|<r\}, p \in \mathcal{H}[0,1] \cap \boldsymbol{Q}(0), q \in \mathcal{H}[0,1] \cap \boldsymbol{Q}(0)$ and suppose

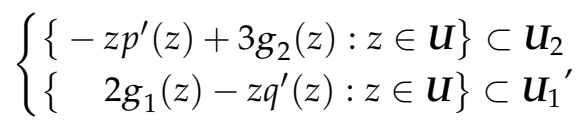


for $g_{1}(z)=g_{2}(z)=z$. It is our intention to prove that

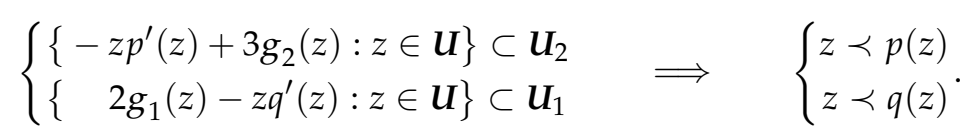

The differential operators in (17) are of the form

$$
\left\{\begin{array}{l}
D_{1}\left[p, g_{2}, \lambda_{1}\right](z)=-z p^{\prime}(z)+3 g_{2}(z) \\
D_{2}\left[g_{1}, q, \lambda_{2}\right](z)=2 g_{1}(z)-z q^{\prime}(z)
\end{array},\right.
$$

with

$$
\left\{\begin{array}{l}
D_{1}\left[r, s, t, \rho, \sigma, \tau, \lambda_{1}(z)\right]=-s+3 \rho \\
D_{2}\left[r, s, t, \rho, \sigma, \tau, \lambda_{2}(z)\right]=2 r-\sigma
\end{array} .\right.
$$

We will use Theorem 1 to prove (18) with $\left\{g_{1}, g_{2}\right\}=\{z, z\}$. We only need to show that the pair of operators $\left(D_{1}, D_{2}\right)$, as given in (19), satisfy the admissibility conditions of Definition 5, namely that $\left(D_{1}, D_{2}\right) \in \boldsymbol{\Psi}_{\left(n_{1}, n_{2}\right)}\left[(0,0),(\mathbf{U}, \mathbf{U}),\left(g_{1}, g_{2}\right)\right]$. According to Definition 5 and (19) this requires showing that

$$
\left\{\begin{aligned}
-z g_{1}^{\prime}(z) / m_{1}+3 g_{2}(\zeta) & \notin \overline{\mathbf{U}}_{2} \\
2 g_{1}(\eta)-z g_{2}^{\prime}(z) / m_{2} & \notin \overline{\mathbf{U}}_{1}^{\prime}
\end{aligned}\right.
$$

when $z \in \mathbf{U}, \zeta \in \partial \mathbf{U}, \eta \in \partial \mathbf{U}, m_{1} \geq 1$ and $m_{2} \geq 1$. This simplifies to the conditions that

$$
\left\{\begin{aligned}
-z / m_{1}+3 \zeta & \notin \overline{\mathbf{U}}_{2} \\
2 \eta-z / m_{2} & \notin \overline{\mathbf{U}}_{1}^{\prime}
\end{aligned}\right.
$$

which are true because of the conditions on the four variables. Hence by Theorem 1 we conclude that

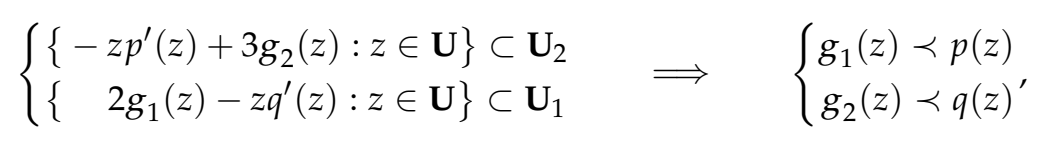

which proves (18).

Example 6. Let $\Omega_{1}=\Omega_{2}=\{z: \operatorname{Re} z>0\}$ and $\lambda_{1}(z)$ be analytic in $\overline{\boldsymbol{U}}$, with $\operatorname{Re} \lambda_{1}(z)>0$. Let $p \in \mathcal{H}\left[1, n_{1}\right] \cap \boldsymbol{Q}(1), q \in \mathcal{H}\left[1, n_{2}\right] \cap \boldsymbol{Q}(1)$ and suppose

$$
\left\{\begin{array}{l}
\left\{-p(z)+g_{2}(z)+\lambda_{1}(z) \cdot z g_{2}^{\prime}(z): z \in \boldsymbol{U}\right\} \subset \Omega_{1} \\
\left\{2 g_{1}(z)+z g_{1}^{\prime}(z)+z^{2} g_{1}^{\prime \prime}(z)-q(z): z \in \boldsymbol{U}\right\} \subset \Omega_{2}^{\prime}
\end{array}\right.
$$

for $g_{1}(z)=g_{2}(z)=(1+z) /(1-z)$. It is our intention to prove that

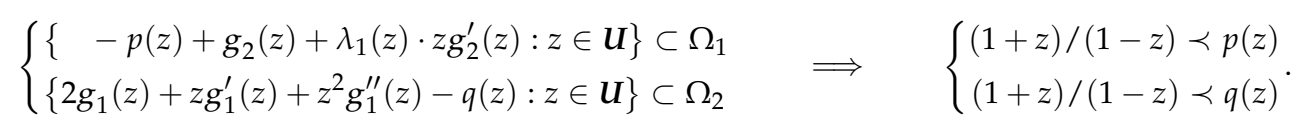

The differential operators in (20) are of the form

$$
\left\{\begin{array}{l}
D_{1}\left[p, g_{2}, \lambda_{1}\right](z)=-p(z)+g_{2}(z)+\lambda_{1}(z) \cdot z g_{2}^{\prime}(z) \\
D_{2}\left[g_{1}, q, \lambda_{2}\right](z)=2 g_{1}(z)+z g_{1}^{\prime}(z)+z^{2} g_{1}^{\prime \prime}(z)-q(z)^{\prime}
\end{array}\right.
$$


with

$$
\left\{\begin{array}{l}
D_{1}\left[r, s, t, \rho, \sigma, \tau, \lambda_{1}(z)\right]=-r+\rho+\lambda_{1}(\zeta) \cdot \sigma \\
D_{2}\left[r, s, t, \rho, \sigma, \tau, \lambda_{2}(z)\right]=2 r+s+t-\rho
\end{array} .\right.
$$

We will use Theorem 1 to prove that if $p$ and $q$ satisfy (20) then they have subordinants $g_{1}$ and $g_{2}$ respectively given by $g_{1}(z)=g_{2}(z)=(1+z) /(1-z)$. We need to show that the pair $\left(D_{1}, D_{2}\right)$ as given in (19) is in the Set of Admissible Operators, i.e., that $\left(D_{1}, D_{2}\right) \in$ $\Psi_{\left(n_{1}, n_{2}\right)}\left[(0,0),\left(h_{1}, h_{2}\right),\left(g_{1}, g_{2}\right)\right]$. We need to show that

$$
\left\{\begin{array}{r}
-g_{1}(z)+g_{2}(\zeta)+\lambda_{1}(\zeta) \cdot \zeta g_{2}^{\prime}(\zeta) \notin \bar{\Omega}_{1} \\
2 g_{1}(\eta)+\eta g_{1}^{\prime}(\eta)+\eta^{2} g_{1}^{\prime \prime}(\eta)-g_{2}(z) \notin \bar{\Omega}_{2}^{\prime}
\end{array}\right.
$$

when $\zeta=e^{i \theta} \in \partial \mathbf{U} \backslash \boldsymbol{E}\left(g_{2}\right), \eta=e^{i \phi} \in \partial \mathbf{U} \backslash \boldsymbol{E}\left(g_{1}\right)$ and $z \in \mathbf{U}$. This follows since

$$
\begin{aligned}
& \operatorname{Re}[\left.-g_{1}(z)+g_{2}\left(e^{i \theta}\right)+\lambda_{1}\left(e^{i \theta}\right) \cdot g_{2}^{\prime}\left(e^{i \theta}\right)\right] \\
&=\operatorname{Re}\left[-\frac{1+z}{1-z}+\frac{1+e^{i \theta}}{1-e^{i \theta}}-\lambda\left(e^{i \theta}\right) \frac{1}{1-\cos \theta}\right]<-\frac{\operatorname{Re} \lambda\left(e^{i \theta}\right)}{1-\cos \theta}<0, \text { and } \\
& \operatorname{Re}\left[2 \frac{1+e^{i \phi}}{1-e^{i \phi}}+e^{i \phi} g_{1}^{\prime}\left(e^{i \phi}\right)+e^{2 i \phi} g_{1}^{\prime \prime}\left(e^{i \phi}\right)-\frac{1+z}{1-z}\right]=\operatorname{Re}\left[\frac{2 e^{i \phi}}{\left(1-e^{i \phi}\right)^{2}}+\frac{4 e^{2 i \phi}}{\left(1-e^{i \phi}\right)^{3}}-\frac{1+z}{1-z}\right] \\
&=\operatorname{Re}\left[\frac{\left(2 e^{i \phi}+2 e^{2 i \phi}\right)\left(1-e^{-i \phi}\right)^{3}}{\left(1-e^{i \phi}\right)^{3}\left(1-e^{-i \phi}\right)^{3}}-\frac{1+z}{1-z}\right]=\operatorname{Re}\left[\frac{4(\sin 2 \phi-2 \sin \phi) i}{\left|1-e^{i \phi}\right|^{6}}-\frac{1+z}{1-z}\right]<0 .
\end{aligned}
$$

Hence by Theorem 1 we conclude that

$$
\left\{\begin{array} { l } 
{ \{ - p ( z ) + g _ { 2 } ( z ) + \lambda _ { 1 } ( z ) \cdot z g _ { 2 } ^ { \prime } ( z ) : z \in \mathbf { U } \} \subset \Omega _ { 1 } } \\
{ \{ 2 g _ { 1 } ( z ) + z g _ { 1 } ^ { \prime } ( z ) + z ^ { 2 } g _ { 1 } ^ { \prime \prime } ( z ) - q ( z ) : z \in \mathbf { U } \} \subset \Omega _ { 2 } }
\end{array} \quad \Longrightarrow \quad \left\{\begin{array}{l}
(1+z) /(1-z) \prec p(z) \\
(1+z) /(1-z) \prec q(z)^{\prime}
\end{array}\right.\right.
$$

if $p$ and $q$ satisfy (20), then $(1+z) /(1-z) \prec p(z)$ and $(1+z) /(1-z) \prec q(z)$.

The definition of the pair of operators $\left(D_{1}, D_{2}\right) \in \boldsymbol{\Psi}_{\left(n_{1}, n_{2}\right)}\left[\left(\lambda_{1}, \lambda_{2}\right),\left(\Omega_{1}, \Omega_{2}\right),\left(g_{1}, g_{2}\right)\right]$, and their dependency on the conditions that $g_{1} \in Q(a)$ and $g_{2} \in Q(b)$ indicates that Theorem 1 depends very heavily on the functions $g_{1}$ and $g_{2}$ behaving very nicely on the boundary of $\mathbf{U}$. If this is not the case or if their behavior on the boundary is unknown, it may still be possible to obtain a variant of the theorem by the following limiting process.

Theorem 2. Let $\lambda_{1}$ and $\lambda_{2}$ be analytic in $\boldsymbol{U}$, let $\left(\Omega_{1}, \Omega_{2}\right)$ be a subset of $\mathbb{C} \times \mathbb{C}$ and let $g_{1}$ and $g_{2}$ be univalent on $\boldsymbol{U}$, with $g_{1}(0)=a$ and $g_{2}(0)=b$. Let $g_{i \rho}(z)=g_{i}(\rho z)$ and $\lambda_{i \rho}(z)=\lambda_{i}(\rho z)$ for $i=1,2$. Let $D_{i}: \mathbb{C}^{7} \rightarrow \mathbb{C}$ for $i=1,2$ and suppose there exists $\rho_{0} \in(0,1)$ such that $\left(D_{1}, D_{2}\right) \in \Psi_{\left(n_{1}, n_{2}\right)}\left[\left(\lambda_{1 \rho}, \lambda_{2 \rho}\right),\left(\Omega_{1}, \Omega_{2}\right),\left(g_{1 \rho}, g_{2 \rho}\right)\right]$ for all $\rho \in\left(\rho_{0}, 1\right)$. If $p \in \mathcal{H}\left[a, n_{1}\right] \cap \boldsymbol{Q}(a)$ and $q \in \mathcal{H}\left[b, n_{2}\right] \cap Q(b)$ have the properties that $D_{1}\left[p, q, \lambda_{1}\right]$ and $D_{2}\left[p, q, \lambda_{2}\right]$ are analytic in $u$ and

$$
\left\{\begin{array}{l}
D_{1}\left[p, g_{2}, \lambda_{1}\right](\boldsymbol{U}) \subset \Omega_{1} \\
D_{2}\left[g_{1}, q, \lambda_{2}\right](\boldsymbol{U}) \subset \Omega_{2}
\end{array}\right.
$$

then $g_{1}(z) \prec p(z)$ and $g_{2}(z) \prec q(z)$.

Proof. If we replace $z$ by $\rho z$ in $p(z), q(z), g_{1}(z), g_{2}(z), \lambda_{1}(z)$ and $\lambda_{2}(z)$ we obtain

$$
\left\{\begin{array}{l}
D_{1}\left[p(\rho z), \rho z p^{\prime}(\rho z), \rho^{2} z^{2} p^{\prime \prime}(\rho z), g_{2}(\rho z), \rho z g_{2}^{\prime}(\rho z), \rho^{2} z^{2} g_{2}^{\prime \prime}(\rho z), \lambda_{1}(\rho z)\right] \subset \Omega_{1} \\
D_{2}\left[g_{1}(\rho z), \rho z g_{1}^{\prime}(\rho z), \rho^{2} z^{2} g_{1}^{\prime \prime}(\rho z), q(\rho z), \rho z q^{\prime}(\rho z), \rho^{2} z^{2} q^{\prime \prime}(\rho z), \lambda_{2}(\rho z)\right] \subset \Omega_{2}{ }^{\prime}
\end{array}\right.
$$

for $z \in \mathbf{U}$. If we set $p_{\rho}(z)=p(\rho z)$ and $q_{\rho}(z)=q(\rho z)$ we obtain 


$$
\left\{\begin{array}{l}
D_{1}\left[p_{\rho}(z), z p_{\rho}^{\prime}(z), z^{2} p_{\rho}^{\prime \prime}(z), g_{2 \rho}(z), z g_{2 \rho}^{\prime}(z), z^{2} g_{2 \rho}^{\prime \prime}(z), \lambda_{1 \rho}(z)\right] \subset \Omega_{1} \\
D_{2}\left[g_{1 \rho}(z), z g_{1 \rho}^{\prime}(z), z^{2} g_{1 \rho}^{\prime \prime}(z), q_{\rho}(z), z q_{\rho}^{\prime}(z), z^{2} q_{\rho}^{\prime \prime}(z), \lambda_{2 \rho}(z)\right] \subset \Omega_{2}
\end{array}\right.
$$

for $z \in \mathbf{U}$. Since $\left(D_{1}, D_{2}\right) \in \boldsymbol{\Psi}_{\left(n_{1}, n_{2}\right)}\left[\left(\lambda_{1 \rho}, \lambda_{2 \rho}\right),\left(\Omega_{1}, \Omega_{2}\right),\left(g_{1 \rho}, g_{2 \rho}\right)\right]$ we can apply Theorem 1 to conclude that $g_{1}(\rho z) \prec p_{\rho}(z)=p(\rho z)$ and $g_{2}(\rho z) \prec q_{\rho}(z)=q(\rho z)$ for $\rho \in\left(\rho_{0}, 1\right)$. If we now let $\rho \rightarrow 1^{-}$, we obtain the results $g_{1}(z) \prec p(z)$ and $g_{2}(z) \prec q(z)$.

\section{Best Subordinants}

In the previous sections we have discussed the problem of finding a set of subordinants for a SSDI. In this section, we discuss a technique for improving that result by finding a set of best subordinants of a SSDI.

Theorem 3. Let $h_{1}$ and $h_{2}$ be analytic in $\boldsymbol{U}, \lambda_{1}$ and $\lambda_{2}$ be analytic in $\overline{\boldsymbol{U}}$, and suppose that the system of simultaneous differential equations

$$
\left\{\begin{array}{l}
D_{1}\left[u, v, \lambda_{1}\right](z)=h_{1}(z) \\
D_{2}\left[u, v, \lambda_{2}\right](z)=h_{2}(z)^{\prime}
\end{array}\right.
$$

has solutions $u=g_{1} \in Q(a)$ and $v=g_{2} \in Q(b)$.

Let $p \in \mathcal{H}\left[a, n_{1}\right] \cap \boldsymbol{Q}(a), q \in \mathcal{H}\left[b, n_{2}\right] \cap \boldsymbol{Q}(b)$ and $\left(D_{1}, D_{2}\right) \in \boldsymbol{\Psi}_{\left(n_{1}, n_{2}\right)}\left[\left(\lambda_{1}, \lambda_{2}\right),\left(h_{1}, h_{2}\right)\right.$, $\left.\left(g_{1}, g_{2}\right)\right]$. If

$$
\left\{\begin{array}{l}
\left\{D_{1}\left[p, g_{2}, \lambda_{1}\right](z): z \in \boldsymbol{U}\right\} \subset h_{1}(\boldsymbol{U}) \\
\left\{D_{2}\left[g_{1}, q, \lambda_{2}\right](z): z \in \boldsymbol{U}\right\} \subset h_{2}(\boldsymbol{U})^{\prime}
\end{array}\right.
$$

then $g_{1}(z) \prec p(z)$ and $g_{2}(z) \prec q(z)$, and the set of functions $\left\{g_{1}, g_{2}\right\}$ is a set of best subordinants of (23).

Proof. Since $\left(D_{1}, D_{2}\right) \in \Psi_{\left(n_{1}, n_{2}\right)}\left[\left(\lambda_{1}, \lambda_{2}\right),\left(h_{1}, h_{2}\right),\left(g_{1}, g_{2}\right)\right]$, from (23) and Theorem 1 we see that the set of functions $\left\{g_{1}, g_{2}\right\}$ form a set of subordinants of SSDI (21). Thus, $g_{1}(z) \prec$ $p(z)$ and $g_{2}(z) \prec q(z)$ for all $p$ and $q$ satisfying (23). On the other hand, the functions $g_{1}$ and $g_{2}$, which are solutions of the System of Simultaneous Differential Equations (20), also satisfy the SSDI (23). Thus, they must be dominant to all subordinants of the system and hence $\left\{g_{1}, g_{2}\right\}$ is a set of best subordinants of system (23). In conclusion, we have the sharp results $g_{1}(z) \prec p(z)$ and $g_{2}(z) \prec q(z)$.

\section{Open Problems}

This article dealt with describing and defining the key terms and elements for finding subordinants of a System of Simultaneous Second-Order Differential Inclusions. We found conditions for finding subordinants for some special cases of such systems. In particular, if $p$ and $q$ are analytic functions satisfying a differential inclusion system of the form

$$
\left\{\begin{array}{l}
D_{1}\left[p, g_{2}, \lambda_{1}\right](\mathbf{U}) \subset \Omega_{1} \\
D_{2}\left[g_{1}, q, \lambda_{2}\right](\mathbf{U}) \subset \Omega_{2}^{\prime}
\end{array}\right.
$$

then we found conditions on the special operators $D_{1}$ and $D_{2}$ so that

$$
\left\{\begin{array} { l } 
{ D _ { 1 } [ p , g _ { 2 } , \lambda _ { 1 } ] ( \mathbf { U } ) \subset \Omega _ { 1 } } \\
{ D _ { 2 } [ g _ { 1 } , q , \lambda _ { 2 } ] ( \mathbf { U } ) \subset \Omega _ { 2 } }
\end{array} \quad \Longrightarrow \quad \left\{\begin{array}{l}
g_{1}(z) \prec p(z) \\
g_{2}(z) \prec q(z)
\end{array} .\right.\right.
$$

The general problem of determining conditions on the operators $D_{1}$ and $D_{2}$, the sets $\Omega_{1}$ and $\Omega_{2}$, and the functions $g_{1}$ and $g_{2}$ so that analytic functions $p$ and $q$ satisfy 


$$
\left\{\begin{array} { l } 
{ D _ { 1 } [ p , q , \lambda _ { 1 } ] ( \mathbf { U } ) \subset \Omega _ { 1 } } \\
{ D _ { 2 } [ p , q , \lambda _ { 2 } ] ( \mathbf { U } ) \subset \Omega _ { 2 } }
\end{array} \quad \Longrightarrow \quad \left\{\begin{array}{l}
g_{1}(z) \prec p(z) \\
g_{2}(z) \prec q(z)^{\prime}
\end{array}\right.\right.
$$

remains an interesting open problem. In addition, the problem of finding the corresponding set of best subordinants of such systems remains an open question.

Author Contributions: Writing—original draft, J.A.A. and S.S.M. Both authors have read and agreed to the published version of the manuscript.

Funding: This research received no external funding.

Institutional Review Board Statement: Not applicable.

Informed Consent Statement: Not applicable.

Data Availability Statement: Not applicable.

Conflicts of Interest: The authors declare no conflict of interest.

\section{References}

1. Miller, S.S.; Mocanu, P.T. Differential Subordinations and Univalent Functions. Michigan Math. J. 1981, 28, 157-171. [CrossRef]

2. Bulboaca, T. Differential Subordinations and Superordinations. Recent Results; Casa Cartii De Stinta: Cluj-Napoca, Romania, 2005.

3. Antonino, J.A.; Miller, S.S. Systems of Simultaneous Differential Inequalities, Inclusions and Subordinations in the Complex Plane. Anal. Math. Phys. 2020, 10, 32. [CrossRef]

4. Miller, S.S.; Mocanu, P.T. Differential Subordinations, Theory and Applications; Marcel Dekker Inc.: New York, NY, USA; Basel, Switzerland, 2000. 